GEOLOGICAL SURVEY CIRCULAR 463

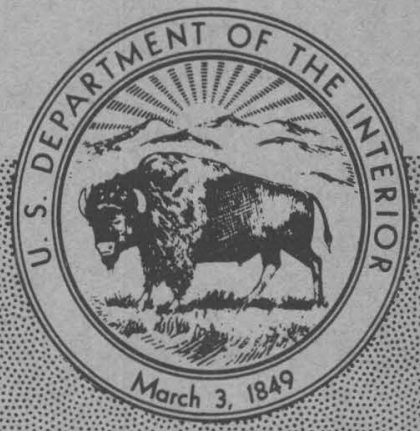

REPORTS AND MAPS OF THE

GEOLOGICAL SURVEY RELEASED

ONLY IN THE OPEN FILES, 1961 



\section{REPORTS AND MAPS OF THE GEOLOGICAL SURVEY RELEASED ONLY IN THE OPEN FILES, 1961}

By Betey A. Weld, Brwin S. Acselotine, and Arthur Johnson 
United States Department of the Interior STEW ART L. UDALI, SBCRBTAKY

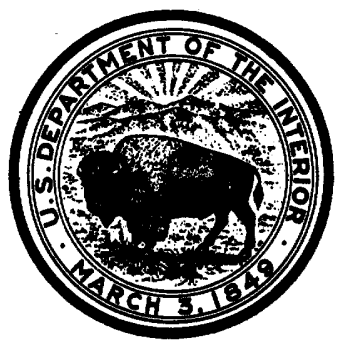

\section{Geological Survey}

THOMAS B. NOLAN, Dirbctor

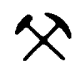

Free on application to the U.S. Geological Survey, Washington 25, D. C. 


\title{
REPORTS AND MAPS OF THE GEOLOGICAL SURVEY RELEASED ONLY IN THE OPEN FILES, 1961
}

\author{
By Betoy A. Weld, Erwin S. Acoelntine, and Arthur Johnson
}

\section{CONT ENTS}

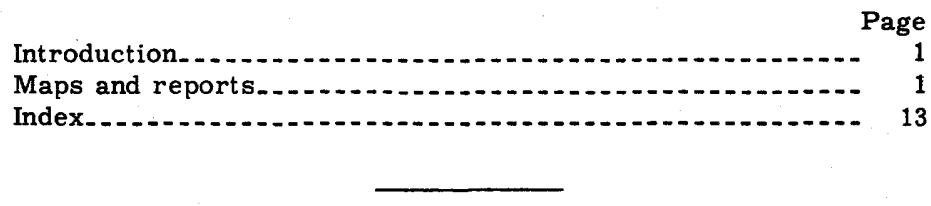

INTRODUCTION

This circular contains a list of the maps and reports released by the Geological Survey in 1961 that are available in open files. They may be consulted at the offices listed beneath each report.

The following circulars list open-file reports and maps released during the years indicated: $56(1946-47)$, 64 (1948), 149 (1949-50), 227 (.1951), 263 (1952), 337 (1953), 364 (1954), 379 (1955), 401 (1956), 403 (1957), 412 (1958), 428 (1959), and 448 (1960).

The Conservation Division has placed 7 reports in open file: 1 on glacier observations in Montana; 2 on waterpower resources in Alaska; 2 reviewing waterpower withdrawals in Oregon; 1 preliminary geologic quadrangle map in Utah;' and 1 report on a coal area in Colorado.

The reports and maps of the Geologic Division contain results of work accomplished by Survey geologists and geophysicists in the United States, Brazil, and
Puerto Rico. These maps and reports are released in preliminary form in order to make information immediately available to persons concerned with exploration for and production of minerals, fuels, and construction materials.

The reports of the Water Resources Division contain information on streamflow, ground water, quality of water, and related geology and hydrology in the United States.

The reports are arranged alphabetically by authors, and each one is preceded by a serial number. In the general index following this list of reports, the serial number is used to identify the report.

The following addresses cited in this circular are Geological Survey libraries: Room 1033, GSA Bldg. (General Services Administration Bldg.), Washington, D. C.; Bldg. 25, Federal Center, Denver, Colo.; and 345 Middlefield Rd., Menlo Park, Calif.

\section{MAPS AND REPORTS}

1. Albee, A. L., and Boudette, E. L., Preliminary geologic map of the Attean quadrangle, Somerset County, Maine: 1 map. Nov. 3, 1961. Room 1033, GSA Bldg., Washington, D. C.; Bldg. 25, Federal Center, Denver, Colo.; 345 Middlefield Rd., Menlo Park, Calif.; Room 1, 270 Dartmouth St., Boston, Mass.; Office of the State Geologist, Dept. of Econ. Devel., State House, Augusta, Maine.

2. Ash, S. R., Geology and ground-water resources of northern Lea County, New Mexico: 53 p., 20 figs. Apr. 14, 1961. Room 279, Geology Bldg., Univ. of New Mexico, Albuquerque, N. Mex.; Room 1242-G, GSA Bldg., Washington, D. C.

3. Bailey, E. H., Mercury in the United States: 1 map. Dec. 26, 1961. Room 1033, GSA Bldg., Washington, D. C.
4. Bailey, R. A., Hot springs and solfotaric areas in the Valles Cáldera, Jemez Mountains, New Mexico: 1 map. Nov. 3, 1961. Room 1033, GSA Bldg., Washington, D. C.; Bldg. 25, Federal Center, Denver, Colo.; 345 Middlefield Rd., Menlo Park, Calif.; Room 468, New Custom House, Denver, Colo.; Room 437, Federal Bldg., Salt Lake City, Utah; Room 602, Thomas Bldg., Dallas, Tex.; Room 1031, Bartlett Bldg., Los Angeles, Calif.; Geol. Survey Field Library, Geology Bldg., Univ, of New Mexico Campus, Albuquerque, N. Mex.; New Mexico Bureau of Mines and Mineral Resources, Socorro, N. Mex.

5. Baker, A. A., Geologic map of the Aspen Grove quadrangle, Utah: 1 map. Jan. 27, 1961. Room 1033, GSA Bldg., Washington, D. C.; Bldg. 25, Federal Center, Denver, Colo.; Room 437, Federal Bldg., Salt Lake City, Utah. 
6. Baker, A. A., Geologic map of southeastern part of Brighton quadrangle, Utah: 1 map. Jan. 27, 1961. Room 1033, GSA Bldg., Washington, D. C.; Bldg. 25, Federal Center, Denver, Colo.; Room 437, Federal Bldg., Salt Lake City, Utah.

7. Baker, A. A., Geologic map of southern part of Heber quadrangle, Utah: 1 map. Jan. 27, 1961. Room 1033, GSA Bldg., Washington, D. C.; Bldg. 25, Federal Center, Denver, Colo.; Room 437, Federal Bldg., Salt Lake City, Utah.

8. Baker, A. A., Geologic map of the Orem quadrangle, Utah: 1 map. Jan. 27, 1961. Room 1033, GSA Bldg., Washington, D. C.; Bldg. 25, Federal Center, Denver, Colo.; Room 437, Federal Bldg., Salt Lake City, Utah.'

9. Baker, A. A., Geologic map of west half of Strawberry Valley quadrangle, Utah: 1 map. Jan. 6, 1961. Room 1033, GSA Bldg., Washington, D. C.; Bldg. 25, Federal Center, Denver, Colo.; Room 437, Federal Bldg., Salt Lake City, Utah.

10. Balsley, J. R., Hill, M. E., and Meuschke, J. L., Total intensity aeromagnetic map of parts of Bolton, Whitehall, Glens Falls, and Fort Ann quadrangles, New York: 1 map. Jan. 27, 1961. Room 1033, GSA Bldg., Washington, D. C.; Bldg. 25, Federal Center, Denver, Colo.; 345 Middlefield Rd., Menlo Park, Calif.; Office of the State Geologist, State Geol. and Nat. History Surveys, State Education Bldg., Albany, N. Y.

11. Balsley, J. R., Hill, M. E., and Meuschke, J. L., Total intensity aeromagnetic map of the Elizabethtown, Paradox Lake, Port Henry, and Ticonderoga quadrangles, New York: 1 map. Jan. 27, 1961. Room 1033, GSA Bldg., Washington, D. C.; Bldg. 25; Federal Center, Denver, Colo.; 345 Middlefield Rd., Menlo Park, Calif.; Office of the State Geologist, State Geol. and Nat. History Surveys, State Education Bldg., Albany, N. $\breve{Y}$.

12. Balsley, J. R., Hill, M. E., and Meuschke, J. L., Total intensity aeromagnetic map of part of the Lowville quadrangle, New York: 1 map. Jan. 27, 1961. Room 1033, GSA Bldg., Washington, D. C.; Bldg. 25, Federal Center, Denver, Colo.; 345 Middlefield Rd., Menlo Park, Calif.; Office of the State Geologist, State Geol. and Nat. History Surveys, State Education Bldg., Albany, N. Y.

13. Balsley, J. R., Hill, M. E., and Meuschke, J. L., Total intensity aeromagnetic map of the McKeever quadrangle, New York: 1 map. Jan. 27, 1961. Room 1033, GSA Bldg., Washington, D. C.; Bldg. 25, Federal Center, Denver, Colo.; 345 Middlefield Rd., Menlo Park, Calif.; Office of the State Geologist, State Geol. and Nat. History Surveys, State Education Bldg., Albany, N. Y.

14. Balsley, J. R., Hill, M. E., and Meuschke, J. L., Total intensity aeromagnetic map of the Number Four quadrangle, New York: 1 map. Jan. 27, 1961. Room 1033, GSA Bldg., Washington, D. C.; Bldg. 25, Federal Center, Denver, Colo.; 345 Middlefield Rd., Menlo Park, Calif.; Office of the State Geologist, State Geol. and Nat. History Surveys, State Education Bldg., Albany, N. Y.
15. Balsley, J. R., Hill, M. E., and Meuschke, J. L., Total intensity aeromagnetic map of the Old Forge quadrangle and part of the West Canada Lakes quadrangle, New York: 1 map. Jan. 27, 1961. Room 1033, GSA Bldg., Washington, D. C.; Bldg. 25, Federal Center, Denver, Colo.; 345 Middlefield Rd., Menlo Park, Calif.; Office of the State Geologist, State Geol. and Nat. History Surveys, State Education Bldg., Albany, N. Y.

16. Balsley, J. R., Hill, M. E., and Meuschke, J. L., Total intensity aeromagnetic map of part of the Port Leyden quadrangle, New York: 1 map. Jan. 27, 1961. Room 1033, GSA Bldg., Washington, D. C.; Bldg. 25, Federal Center, Denver, Colo.; 345 Middlefield Rd., Menlo Park, Calif.; Office of the State Geologist, State Geol. and Nat. History Surveys, State Education Bldg., Albany, N. Y.

17. Balsley, J. R., Rossman, D. L., and Hill, M. E., Total intensity aeromagnetic map of the Big Moose quadrangle, New York: 1 map. Jan. 27, 1961. Room 1033, GSA Bldg., Washington, D. C.; Bldg. 25, Federal Center, Denver, Colo.; 345 Middlefield Rd., Menlo Park, Calif.; Office of the State Geologist, State Geol. and Nat. History Surveys, State Education Bldg., Albany, N. Y.

18. Barnell, R. L., and DeLuca, F. A., Ground-water levels in Long Island, New York, 1958-1959: 66 p., 6 figs. Oct. 6, 1961. 1505 Kellum Place, Mineola, N. Y.; Room 1242-G, GSA Bldg., Washington, D. C.

19. Barnes, Harley, Houser, F: N., and Poole, F. G., Geologic map of Oak Spring quadrangle, Nye County, Nevada: 1 map with explanation. Aug. 23, 1961. Room 1033, GSA Bldg., Washington, D. C.; Bldg. 25, Federal Center, Denver, Colo.; 345 Middlefield Rd., Menlo Park, Calif.; Room 232, Appraisers Bldg., San Francisco, Calif.; Room 1031, Bartlett Bldg., Los Angeles, Calif.; Room 437, Federal Bldg., Salt Lake City, Utah; Room 279, Geology Bldg., Univ. of New Mexico, Albuquerque, N. M.; Mercury, Nev.; Library, Mackay School of Mines, Univ. of Nevada, Reno, Nev.

20. Bedinger, M. S., and Sniegocki, R. T., Groundwater problems in Missouri: 21 p., 4 figs. Dec. 20, 1961. 217 Main St., 2d Floor, Little Rock, Ark.; Room 1242-G, GSA Bldg., Washington, D. C.

21. Bell, K. G., Rhoden, V. C., McDonald, R. L., and Bunker, C. M., Utilization of gamma-ray logs by the U.S. Geological Survey, 1949-1953. 89 p., 24 figs., 1 table. Apr. 24, 1961 . Room 1033, GSA Bldg., Washington, D. C.; BIdg. 25, Federal Center, Denver, Colo.; 345 Middlefield Rd., Menlo Park, Calif.; Room 468, New Custom House, Denver, Colo.; Room 232, Appraisers Bldg., San Francisco, Calif.; Koom 1031 , Bartlett Bldg., Los Angeles, Calif.; Koom 503, Cordova Bldg., Anchorage, Alaska; South 157 Howard St., Spokane, Wash.; Room 437, Federal Bldg., Salt Lake City, Utah; Room 602, Thomas Bldg., Dallas, Tex.; U.S. Atómic Energy Commission, Grand Junction, Colo. 
22. Bjorklund, L. J., Semiannual report of water levels in selected observation wells in Utah, October 1961: 1 p., 3 figs. Oct. 9, 1961. Room 503-A, Federal Bldg., Salt Lake City, Utah; Room 1242G, GSA Bldg., Washington, D. C.

23. Bradley, Edward, and Petersen, R. G., Records and logs of selected wells and test holes, chemical analyses of water, and water levels in observation wells in southeastern New Hampshire: 220 p., 5 figs. Nov. 16, 1961 . Room 432, Federal Bldg., Trenton, N. J.; Room 1242-G, GSA Bldg., Washington, D. C.

24. Briggs, R. P., Preliminary geologic map of Puerto Rico and adjacent islands: 1 map. Oct. 9, 1961. Room 1033, GSA Bldg., Washington, D. C.; Library, Dept. of Industrial Research, Puerto Rico Devel. Admin., Franklin D. Roosevelt Avenue and Soldado Rafael Lamar St., Hato Rey, Puerto Rico.

25. Bromery, R. W., and Gilbert, F. P., Aeromagnetic maps of the Attean quadrangle and part of the Sandy Bay quadrangle, Somerset County, Maine: 2 maps. May 23, 1961. Room 1033, GSA Bldg., Washington, D. C.; Room 1, 270 Dartmouth St., Boston, Mass.; Office of the State Geologist, Dept. of Econ. Devel., State House, Augusta, Maine.

26. Bromery, R. W., and Natoff, N. C., Aeromagnetic maps of the Bingham quadrangle, Somerset County, Maine, and The Forks quadrangle, Piscataquis and Somerset Counties; Maine: 2 maps. May 23, 1961. Room 1033, GSA Bldg., Washington, D. C.; Room 1, 270 Dartmouth St., Boston, Mass.; Office of the State Geologist, Dept. of Econ. Devel., State House, Augusta, Maine.

27. Bunker, C. M., Gamma-radioactivity investigations at the Nevada Test Site, Nye and Lincoln Counties, Nevada: 95 p., 52 figs., 2 tables. June 29, 1961. Room 1033, GSA Bldg., Washington, D. C.; Bldg. 25, Federal Center, Denver, Colo.; 345 Middlefield Rd., Menlo Park, Calif.; Room 232, Appraisers Bldg., San Francisco, Calif.; Room 1031, Bartlett Bldg., Los Angeles, Calif.; Room 437, Federal Bldg., Salt Lake City, Utah; Room 279, Geology Bldg., Univ. of New Mexico, Albuquerque, N. Mex.; Library, Mackay School of Mines, Univ. of Nevada, Reno, Nev.

28. Busch, W. F., and Shaw, E. C., Floods in Pennsylvania, frequency and magnitude: 231 p., 2 pls., 11 figs. Apr. 24, 1961. Room 490, Education Bldg., Harrisburg, Pa.; 4th floor, Victory Bldg., 9th St. and Liberty Ave., Pittsburgh, Pa.; Room 2236, GSA Bldg., Washington, D. C.

29. Butler, A. P., Jr., Finch, W. I. and Twenhofel, W. S., Epigenetic uranium deposits in the United States: 1 map. Dec. 26, 1961. Room 1033, GSA Bldg., Washington, D. C.

30. Cheney, T. M., Wolcott, D. E., and Schilling, F. A., Jr., Preliminary geologic map of the Stewart Flat quadrangle, Caribou County, Idaho: 1 map. May 17, 1961. Room 1033, GSA Bldg., Washington, D. C.; Bldg. 25, Federal Center, Denver,
Colo.; 345 Middlefield Rd., Menlo Park, Calif.; South 157 Howard St., Spokane, Wash.; Room 437, Federal Bldg., Salt Lake City, Utah.

31. Chidester, A. H., and Shride, A. F., Asbestos in the United States: 1 map. Dec. 26, 1961. Room 1033, GSA Bldg., Washington, D. C.

32. Chidester, A. H., and Worthington, H. W., Talc and soapstone in the United States: 1 map. Dec. 26, 1961. Room 1033, GSA Bldg., Washington, D. C.

33. Colbert, J. L., Review of waterpower withdrawals in McKenzie River Basin, Oregon: 25 p., 1 fig. July 15, 1961. Room 1033, GSA Bldg., Washington, D. C.; 1002 N. E. Holladay St., Portland, Oreg.

34. Cooper, J. R., Bismuth in the United States: 1 map. Dec. 26, 1961. Room 1033, GSA Bldg., Washington, D. C.

35. Crandell, D. R., Surficial geology of the Orting quadrangle, Washington: 1 map and explanation. Jan. 27, 1961. Room 1033, GSA Bldg., Washington, D. C.; Bldg. 25, Federal Center, Denver, Colo.; 345 Middlefield Rd., Menlo Park, Calif.; South 157 Howard St., Spokane, Wash.; 3020 South 38th St., Tacoma, Wash.; Div. of Mines and Geology, Room 335, General Admin. Bldg., Olympia, Wash.

36. Crandell, D. R., Surficial geology of the Sumner quadrangle, Washington: 1 map and explanation. Jan. 27, 1961 . Room 1033, GSA Bldg., Washington, D. C.; Bldg. 25, Federal Center, Denver, Colo.; 345 Middlefield Rd., Menlo Park, Calif.; South 157 Howard St., Spokane, Wash.; 3020 South 38 th St., Tacoma, Wash.; Div. of Mines and Geology, Room 335, General Admin. Bldg., Olympia, Wash.

37. Crandell, D. R., Surficial geology of the Wilkeson quadrangle, Washington: 1 map and explanation. Jan. 27, 1961. Room 1033, GSA Bldg., Washington, D. C.; Bldg. 25, Federal Center, Denver, Colo.; 345 Middlefield Rd., Menlo Park, Calif.; South 157 Howard St., Spokane, Wash.; 3020 South 38th St., Tacoma, Wash.; Div. of Mines and Geology, Room 335, General Admin. Bldg.. Olympia, Wash.

38. Crittenden, M. D., Jr., Isostatic deformation of Bonneville shorelines: 4 p., 2 figs. Sept. 12, 1961. Room 1033, GSA Bldg., Washington, D. C.; Bldg. 25, Federal Center, Denver, Colo.; 345 Middlefield Rd., Menlo Park, Calif.; Room 437, Federal Bldg., Salt Lake City, Utah; Room 468, New Custom House, Denver, Colo.

39. Crittenden, M. D., Jr., and Pavlides, Louis, Manganese in the United States: 1 map. Dec. 26, 1961. Room 1033, GSA Bldg., Washington, D. C.

40. de Witt, Wallace, Jr., Geology of the Michigan basin with reference to subsurface disposal of radioactive wastes: 100 p., 20 figs. Apr. 22, 1961. Room 1033, GSA Bldg., Washington, D. C.; Bldg. 25, Federal Center, Denver, Colo.; 345 Middlefield Rd., Menlo Park, Calif.; Room 468, New 
Custom House, Denver, Colo.; Room 602, Thomas Bldg., Dallas, Tex.; Room 1031, Bartlett Bldg., Los Angeles, Calif.; Room 232, Appraisers Bldg., San Francisco, Calif.; Room 437, Federal Bldg., Salt Lake City, Utah; South 157 Howard St., Spokane, Wash.; Room 279, Geology Bldg., Univ. of New Mexico, Albuquerque, N. Mex;; State Geol. Survey Div., Dept. of Conservation, Lansing, Mich.

41. Dickey, D. D., and Emerick, W. L., Interim report on geologic investigations of the U12b tunnel system, Nevada Test Site, Nye County, Nevada: 13 p., 2 figs., 2 tables. Dec. 5; 1961. Room 1033, GSA Bldg., Washington, D. C.; Bldg. 25, Federal Center, Denver, Colo.; 345 Middlefield Rd., Menlo Park, Calif.; Mercury, Nev.; Room 279, Geology Bldg., Univ. of New Mexico, Albuquerque, N. .Mex.; Room 232, Appraisers Bldg., San Francisco, Calif.; Room 1031, Bartlett Bldg., Los Angeles, Calif.; Room 437, Federal Bldg., Salt Lake City, Utah; Library, Mackay School of Mines, Univ, of Nevada, Reno, Nev.

42. Dunham, R. J., Geology of uranium in the Chadron area, Nebraska and South Dakota: 243 p., 1 pl., 36 figs., 9 tables. June 14, 1961. Room 1033, GSA Bldg., Washington, D. C.; Bldg. 25, Federal Center, Denver, Colo.

43. Dyer, H. B., Ground-water conditions during 1960 at the Marine Corps Base, Twentynine Palms, California: 32 p., 7 figs. Mar. 1, 1961. 221 Redondo Ave., Long Beach, Calif.; Room 8024, Federal and U.S. Court House Bldg., 650 Capitol Ave., Sacramento, Calif.; Room 1242-G, GSA Bldg., Washington, D. C.

44. Ericson, D. W., Floods in Wisconsin, magnitude and frequency: 109 p., 19 figs. July 7, 1961. Room 699, State Office Bldg., Madison, Wis.; Room 2236, GSA Bldg., Washington, D. C.

45. Ericson, D. W., Wisconsin River near Dekorra, Wis., flood-flow characteristics at proposed bridge site on the Wisconsin freeway in Columbia County: 13 p., 8 figs. June 26, 1961. Room 699, State Office Bldg., Madison, Wis.; Room 2236, GSA Bldg., Washington, D. C.

46. Espenshade, G. H., Pyrophyllite and kyanite and related minerals in the United States: 1 map. Dec. 26, 1961. Room 1033, GSA Bldg., Washington, D. C.

47. Evenson, R. E., Availability of ground water near Pedernales, California: 52 p., 2 figs. Sept. 28, 1961. Room 8024, Federal and U.S. Court House Bldg., 650 Capitol Ave., Sacramento, Calif.; Room 1242-G, GSA Bldg., Washington, D. C.

48. Evenson, R. E., Ground-water conditions, Naval Missile Facility, Point Arguello, California, June 30, 1960: 26 p., 3 figs. Mar. 31, 1961. Room 8024, Federal and U.S. Court House Bldg., 650 Capitol Ave., Sacramento, Calif.; Room 1242-G, GSA Bldg., Washington, D. C.
49. Feltis, R. D., Semiannual report of water levels in selected observation wells in Utah, April 1961: 1 p., 3 figs. Apr. 5, 1961. Room 503-A, Federal Bldg., Salt Lake City, Utah: Room 1242-G, GSA Bldg., Washington, D. C.

50. Feulner, A. J., Data on wells at Ladd Air Force Base, Alaska, 1960: 41 p., 2 figs. Mar. 28, 1961. Room 501, Cordova Bldg., Anchorage, Alaska; Room 1242-G, GSA Bldg., Washington, D. C.

51. Fischer, R. P., Vanadium in the United States: 1 map. Dec. 26, 1961. Room 1033, GSA Bldg., Washington, D. C.

52. Frazier, A. H., Care and rating of current meters: 28 p., 4 figs. Nov. 30, 1961. Room 1033, GSA Bldg., Washington, D. C.

53. Gardner, L. S., Preliminary geologic map, columnar sections and trench sections of the Irwin quadrangle, Caribou and Bonneville Counties, İaho, and Lincoln and Teton Counties, Wyoming: 7 sheets. Apr. 24, 1961. Poom 1033, GSA Bldg., Washington, D. C.; Bldg. 25, Federal Center, Denver, Colo,; 345 Middlefield Rd., Menlo Park, Calif.; Idaho Bureau of Mines and Geology, Univ. of Idaho, Moscow, Idaho.

54. Gaskill, D. L., and Horn, G. H., Northeast Rangely coal area, Rio Blanco and Moffat Counties, Colorado: 49 p., 2 pls., 3 figs. Dec. 29, 1961. Room 1033, GSA Bldg., Washington, D. C.; Room 1329, Bldg. 25, Federal Center, Denver, Colo.; Room 468, New Custom House, Denver, Colo.

55. Gibbons, A. B., Hinrichs, E. N., Dickey, D. D., McKeown, F. A., Poole, F. G., and Houser, F. N., Engineering geology of test sites in granite and dolomite at Gold Meadows, Climax, and Dolomite Hill, Nevada Test Site, Nye County, Nevadapreliminary report: 42 p., 5 figs., 6 tables. Jan. 30, 1961. Room 1033, GSA Bldg., Washington, D. C.; Bldg. 25, Federal Center, Denver, Colo.; 345 Middlefield Rd., Menlo Park, Calif.

56. Gildersleeve, Benjamin, Magnesite and brucite in the United States: 1 map. Dec. 26, 1961. Room 1033, GSA Bldg., Washington, D. C.

57. Griffin, M. S., and Clark, E. N., Bibliography of technical reports, articles, and memoranda published or otherwise released by the Ground Water Branch during the calendar year, 1960: 38 p. Jan. 30, 1961. Room 1242-G, GSA Bldg., Washington, D. C.

58. Harshbarger, J. W., and others, Maps of Navajo Indian Reservation and vicinity, Arizona: 9 figs. June 27, 1961. Geology Bldg., Univ. of Arizona Campus, Tucson, Ariz.: Room 1242-G, GSA Bldg., Washington, D. C.

59. Hart, D. L., Jr., Ground water in the alluvium of Beaver Creek basin, Oklahoma: 13 p., 1 fig. Jan. 12 , 1961. Bldg. 901, Univ. of Oklahoma, North Campus, 
Norman, Okla.; Room 1242-G, GSA Bldg., Washington, D. C.

60. Hazlewood, R. M., Interim report on seismic velocities of the Oak Spring formation, U12e and U12b tunnel systems, Nevada Test Site, Nye County, Nevada: 12 p., 4 figs., 1 table. Oct. 20, 1961. Room 1033, GSA Bldg., Washington, D. C.: Bldg. 25, Federal Center, Denver, Colo.; 345 Middlefield Rd., Menlo Park, Calif.; Room 232, Appraisers Bldg., San Francisco, Calif.; Room 1031, Bartlett Bldg., Los Angeles, Calif.; Room 437, Federal Bldg., Salt Lake City, Utah; Room 279, Geology Bldg., Univ. of New Mexico, Albuquerque, N. Mex.; Mercury, Nev.; Library, Mackay School of Mines, Univ. of Nevada, Reno, Nev.

61. Hely, A. G., The surface-water supply of the lower Colorado River area: 16 p. Nov. 17, 1961. 16 W. 2d St., Yuma, Ariz.; Room 1242-G, GSA Bldg., Washington, D. C.

62. Hendrickson, G. E., Investigations of ground-water resources of the lower Colorado River: $7 \mathrm{p}$. Nov. 17, 1961.16 W. 2d St., Yuma, Ariz.; Room 1242-G, GSA Bldg., Washington, D. C.

63. Hill, D. P., and Jacobson, J. J., Gravity survey in the western Snake River Plain, Idaho-a progress report: 20 p., 4 figs., 2 sheets. June 14, 1961. Room 1033, GSA Bldg., Washington, D. C.; Bldg. 25, Federal Center, Denver, Colo.; 345 Middlefield Rd., Menlo Park, Calif.; Idaho Bureau of Mines and Geology, Univ. of Idaho, Moscow, Idaho.

64. Hoare, J. M., Preliminary geology along the lower Yukon River, Alaska: 1 map. Sept. 18, 1961. Room 1033, GSA Bldg., Washington, D. C.; Bldg. 25, Federal Center; Denver, Colo.; 345 Middlefield Rd., Menlo Park, Calif.; Room 503, Cordova Bldg., Anchorage, Alaska; Brooks Memorial Bldg., College, Alaska; Room 117, Federal Bldg., Juneau, Alaska; State Div. of Mines and Minerals, State Capitol Bldg., Juneau, Alaska; South 157 Howard St., Spokane, Wash.; Room 232, Appraisers Bldg., San Francisco, Calif.; Room 1031, Bartlett Bldg., Los Angeles, Calif.; Room 468, New Custom House, Denver, Colo.; Room 602, Thomas Bldg., Dallas, Tex.

65. Hollowell, J. R., Ground water in the alluvium of Elk Creek basin, Oklahoma: 27 p., 6 figs. Aug. 11, 1961. Bldg. 901, Univ. of Oklahoma, North Campus, Norman, Okla.; Room 1242-G, GSA Bldg., Washington, D. C.

66. Hollowell, J. R., Ground water in the alluvium of Otter Creek basin, Oklahoma: 21 p., 7 figs. Aug. 21, 1961. Bldg. 901, Univ. of Oklahoma, North Campus, Norman, Okla.; Room 1242-G, GSA Bldg., Washington, D. C.

67. Hollowell, J. R., Ground water in the vicinity of Roosevelt, Oklahoma: 9 p., 3 figs. Nov. 16, 1961. Bldg. 901, Univ. of Oklahoma, North Campus, Norman, Oklahoma.; Room 1242-G, GSA Bldg., Washington, D. C.
68. Hood, J. W., Water wells in Frenchman and Yucca Valleys, Nevada Test Site, Nye County, Nevada: 58 p., -13 figs. Aug. 8, 1961. Room 1033, GSA Bldg., Washington, D. C.; Bldg. 25, Federal Center, Denver, Colo.; 345 Middlefield Rd., Menlo Park, Calif.; Room 232, Appraisers Bldg., San Francisco, Calif.; Room 1031, Bartlett Bldg., Los Angeles, Calif.; Room 437, Federal Bldg., Salt Lake City, Utah; Room 279, Geology Bldg., Univ. of New Mexico, Albuquerque, N. Mex.; Library, Mackay School of Mines, Univ. of Nevada, Reno, Nev.

69. Houser, F. N., Lithologic logs of three exploration core holes, U15b area, Climax Stock, Nevada Test Site, Nye County, Nevada: 67 p., 1 fig., 1 table. Sept. 12, 1961. Room 1033, GSA Bldg., Washington, D. C.; Bldg. 25, Federal Center, Denver, Colo.; 345 Middlefield Rd., Menlo Park, Calif.; Room 232, Appraisers Bldg., San Francisco, Calif.; Room 1031, Bartlett Bldg., Los Angeles, Calif.; Room 437, Federal Bldg., Salt Lake City, Utah; Room 279, Geology Bldg., Univ. of New Mexico, Albuquerque, N. Mex.; Library, Mackay School of Mines, Univ, of Nevada, Reno, Nev.

70. Houser, F. N., Davis, R. E., and Emerick, W. L., Geologic reconnaissance of granitic intrusive masses at Gold Meadows, Tem Piute and Trappman's Camp, Lincoln and Nye Counties, Nevada, and comparison with the Climax Stock at the Nevada Test Site: 15 p., 1 fig., 2 tables. Oct. 20, 1961. Room 1033, GSA Bldg., Washington, D. C.; Bldg. 25, Federal Center, Denver, Colo.; 345 Middlefield Rd., Menlo Park, Calif.; Room 232, Appraisèrs Bldg., San Francisco, Calif.; Room 1031, Bartlett Bldg., Los Angeles, Calif.; Room 437, Federal Bldg., Salt Lake City, Utah; Room 279, Geology Bldg., Univ. of New Mexico, Albuquerque, N. Mex.; Mercury, Nev.; Library, Mackay School of Mines, Univ. of Nevada, Reno, Nev.

71. Hughes, G. H., Determining evaporation from the Salton Sea by the energy-budget method: 12 p., 1 fig. Nov. 17, 1961. 16 W. 2d St., Yuma, Ariz.; Room 1242-G, GSA Bldg., Wastington, D. C.

72. Indermuhle, V. C., Preliminary report on the waterpower resources of Snow River, Nellie Juan Lake, and Lost Lake, Kenai Peninsula, Alaska: 55 p., 3 pls., 11 figs., 9 tables. July 10 , 1961. Room 1033, GSA Bldg., Washington, D. C.; Room 503, Cordova Bldg., Anchorage, Alaska; Room 111, Federal Bldg., Juneau, Alaska; Wright Bldg., Palmer, Alaska; Room 1031, Bartlett Bldg., Los Angeles, Calif.; Room 232, Appraisers Bldg., San Francisco, Calif.; Room 468, New Custom House, Denver, Colo.; Room 602, Thomas Bldg., Dallas, Tex.; South 157 Howard St., Spokane, Wash.; Room 244, Federal Bldg., Tacoma, Wash.

73. Irelan, Burdge, Quality of water considerations, lower Colorado River area: 7 p. Nov. 17, 1961. 16 W. 2d St., Yuma, Ariz.; Room 1242-G, GSA Bldg., Washington, D. C. 
74. Jackson, B. L., Potential waterpower of Lake Chakachamna, Alaska: 20 p., 4 figs., 2 tables. July 10, 1961 . Room 1033, GSA Bldg., Washington, D. C.; Room 503, Cordova Bldg., Anchorage, Alaska; Room 111, Federal Bldg., Juneau, Alaska; Wright Bldg., Palmer, Alaska; Room 1031, Bartlett Bldg., Los Angeles, Calif.; Room.232, Appraisers Bldg., San Francisco, Calif.; Room 468, New Custom House, Denver, Colo.; Room 602, Thomas Bldg., Dallas, Tex.; South 157 Howard St., Spokane, Wash.; Room 244, Federal Bldg., Tacoma, Wash.

75. James, H. L., and Wier, K. L., Carter Creek iron deposit, Madison and Beaverhead Counties, Montana: 2 sheets. Mar. 14, 1961. Room 1033, GSA Bldg., Washington, D. C.; Bldg. 25, Federal Center, Denver, Colo.; 345 Middlefield Rd., Menlo Park, Calif.; South 157 Howard St., Spokane, Wash.; Montana Bureau of Mines and Geology, Montana School of Mines, Butte, Mont.

76. James, H. L., and Wier, K. L., Geologic map of the Kelly iron deposit, Madison County, Montana: 2 sheets. Mar. 14, 1961. Room 1033, GSA Bldg., Washington, D. C.; Bldg. 25, Federal Center, Denver, Colo.; 345 Middlefield Rd., Menlo Park, Calif.; South 157 Howard St., Spokane, Wash.; Montana Bureau of Mines and Geology, Montana School of Mines, Butte, Mont.

77. Jenkins, C. T., Preliminary report on floods on Boulder Creek below Boulder, Colorado: 25 p., 1 map, 6 figs. Nov. 30, 1961. Bldg. 25, Federal Center, Denver, Colo.; Room 1033, GSA Bldg., Washington, D. C.

78. Johnson, Arthur, Glacier observations, Glacier National Park, Montana, 1960: 15 p., 1 fig. May 8, 1961. Room 1033, GSA Bldg., Washington, D. C.; Room 244, Federal Bldg., Tacoma, Wash.; South 157 Howard St., Spokane, Wash.; Room 409, Federal Bldg., Helena, Mont.; Room 437, Federal Bldg., Salt Lake City, Utah; Room 307, Federal Office Bldg., Omaha, Nebr.; Glacier National Park, West Glacier, Mont.

79. Johnston, P. M., Basic data, ground-water resources and geology of Washington, D. C., and vicinity: 320 p. Aug. 18, 1961. Room 1242-G, GSA Bldg., Washington, D. C.

80. Jones, P. H., Hydrology of radioactive waste disposal at the Idaho Chemical Processing Plant National Reactor Testing Station, Idaho: 8 p., 4 figs. Feb. 1, 1961. 914 Jefferson St., Boise, Idaho: Room 1242-G, GSA Bldg., Washington, D. C.

81. Kachadoorian, Reuben, Geologic aspects of the November 1960 high-explosives test and the Project Chariot Site, northwestern Alaska: 15 p., 1 pl., 4 figs. Sept. 22, 1961 . Room 1033, GSA Bldg., Washington, D. C.; Bldg. 25, Federal Center, Denver, Colo.; 345 Middlefield Rd., Menlo Park, Calif.; Room 503, Cordova Bldg., Anchorage, Alaska; Brooks Memorial Bldg., College, Alaska; Room 117, Federal Bldg., Juneau, Alaska; State Div: of Mines and Minerals, State Capitol Bldg., Juneau, Alaska; South 157 Howard St., Spokane, Wash.; Room 232, Appraisers Bldg.,
San Francisco, Calif.; Room 1031, Bartlett Bldg., Los Angeles, Calif.; Room 468, New Custom House, Denver, Colo.; Room 602, Thomas Bldg., Dallas, Tex.; Room 279, Geology Bldg., Univ, of New Mexico, Albuquerque, N. Mex.

82. Kane, M. F., and Peterson, D. L., Preliminary interpretation of gravity data in west-central Maine: 10 p., 1 pl. July 28, 1961 . Room 1033, GSA Bldg., Washington, D. C.; Room 1, 270 Dartmouth St., Boston, Mass.; Office of the State Geologist, Dept. of Econ. Devel., State House, Augusta, Maine.

83. Kennedy, V. C., Geochemical studies of mineral deposits in the Lisbon Valley area, San Juan County, Utah: 157 p., 15 figs., 34 tables. Aug. 23, 1961. Room 1033, GSA Bldg., Washington, D. C.

84. Kinkel, A. R., Jr., and Peterson, N. P., Copper in the United States: 1 map. Dec. 26, 1961. Room 1033, GSA Bldg., Washington, D. C.

85. Koschmann, A. H., and Bergendahl, M. H., Gold in the United States: 1 map. Dec. 26, 1961. Room 1033, GSA Bldg., Washington, D. C.

86. Krieger, R. A., Chemical quality conditions of the Green River at Munfordville, Kentucky, October 1956-September 1961: 5 p., 5 figs. Nov. 3, 1961. 2822 East Main St., Columbus, Ohio; Room 1242D, GSA Bldg., Washington, D. C.

87. Lemmon, D. M., and Tweto, Ogden, Tungsten in the United States: 1 map. Dec. 26, 1961. Room 1033, GSA Bldg., Washington, D. C.

88. Liesch, B. A, and Norvitch, R. F., Map of the Chisholm-Dewey Lake area, Minnesota, showing surficial geology and contours on the piezometric surface: 1 map. June 8, 1961 . Room 1002, P. O. Bldg., St. Paul, Minn.; Room 1242-G, GSA Bldg., Washington, D. C.

89. Loney, R. A., Structure and stratigraphy of the Pybus-Gambier area, Alaska: 200 p., 5 pl.; 56 figs. Sept. 12, 1961. Room 1033, GSA Bldg., Washington, D. C.; 345 Middlefield Rd., Menlo Park, Calif.; Room 503, Cordova Bldg.. Anchorage, Alaska; Brooks Memorial Bldg., College, Alaska.

90. Love, J. D., and Hoover, Linn, A summary of the geology of sedimentary basins of the United States, with reference to the disposal of radioactive wastes: 89 p., 1 fig. and map index. Feb. 28, 1961. Room 1033, GSA Bldg., Washington, D. C.; Bldg. 25, Federal Center, Denver, Colo.; 345 Middlefield Rd., Menlo Park, Calif.; Room 468, New Custom House, Denver, Colo.; Room 602, Thomas Bldg., Dallas, Tex.; Room 232, Appraisers Bldg., San Francisco, Calif.; Room 1031, Bartlett Bldg., Los Angeles, Calif.; Room 437, Federal Bldg., Salt Lake City, Utah; Room 279, Geology Bldg., Univ. of New Mexico, Albuquerque, N. Mex.

91. McDonald, C. C., Investigation of the water resources of the lower Colorado River arpe: 5 p. Mar. 10, 
1961. 16. W. 2d St., Yuma, Ariz.; Room 1242-G; GSA Bldg., Washington, D. C.

92. McDonald, C. C., Studies of the hydrology of the lower Colorado River area: 8 p. Nov. 17, 1961, 16 W. 2d St., Yuma, Ariz,; Room 1242-G, GSA Bldg., Washington, D. C.

93. Mckeown, F. A., and Dickey, D. D., Interim report on geologic investigations of the U12e tunnel system, Nevada Test Site, Nye County, Nevada: 16 p., 8 figs., 6 tables. June 14, 1961. Room 1033, GSA Bldg., Washington, D. C.; Bldg. 25, Federal Center, Denver, Colo.; 345 Middlefield Rd., Menlo Park, Calif.; Room 437, Federal Bldg., Salt Lake City, Utah; Room 232, Appraisers Bldg., San Francisco, Calif.; Room 1031, Bartlett Bldg., Los Angeles, Calif.; Room 279, Geology Bldg., Univ. of New Mexico, Albuquerque, N: Mex.; Library, Mackay School of Mines, Univ. of Nevada, Reno, Nev.

94. McKnight, E. T., Newman, W. L., and Heyl, A. V., Jr., Lead in the United States: 1 map. Dec. 26, 1961. Room 1033, GSA Bldg., Washington, D. C.

95. McKnight, E. T., Newman, W. L., and Heyl, A. V., $\mathrm{Jr}$. , Zinc in the United States: 1 map. Dec. 26, 1961. Room 1033, GSA Bldg., Washington, D. C.

96. Merritt, P. M., Water levels in observation wells in Santa Barbara County, California, in 1960: 51 p., 9 figs. Mar. 13, 1961. Room 8024, Federal and U.S. Court House Bldg., 650 Capitol Ave., Sacramento, Calif.; Room 1242-G, GSA Bldg., Washington, D. C.

97. Metzger, Donald G., Geohydrologic investigations of the lower Colorado River valley: 8 p. Nov. 17 , 1961. 16 W. 2d St.. Yuma, Ariz.; Room 1242-G, GSA Bldg., Washington, D. C.

98. Miesch, A. T., Geochemistry of the Frenchy Incline uranium deposit, San Miguel County, Colorado: 112 p., 25 figs., 8 tables. July 28, 1961. Room 1033, GSA Bldg., Washington, D. C.; Bldg. 25, Federal Center, Denver, Colo.; 345 Middlefield Rd., Menlo Park, Calif,; Room 437, Federal Bldg., Salt Lake City, Utah; Room 468, New Custom House, Denver, Colo.; U.S. Atomic Energy Commission, Grand Junction, Colo.

99. Miller, D. J., Geology of the Katalla district, Gulf of Alaska Tertiary province, Alaska: 2 sheets. June 2, 1961. Room 1033, GSA Bldg., Washington, D. C.; Bldg. 25, Federal Center, Denver, Colo.; 345 Middlefield Rd., Menlo Park, Calif.; Brooks Memorial Bldg., College, Alaska; Room 117, Federal Bldg., Juneau, Alaska; State Div. of Mines and Minerals, State Capitol Bldg., Juneau, Alaska; Room 503, Cordova Bldg., Anchorage, Alaska; Room 232, Appraisers Bldg., San Francisco, Calif.; Room 1031, Bartlett Bldg., Los Angeles, Calif.; South 157 Howard St., Spokane, Wash.; Room 468, New Custom House, Denver, Colo.; Room 602, Thomas Bldg., Dallas, Tex.
100. Miller, D. J., Geolugy of the Lituya district, Gulf of Alaska Tertiary province, Alaska: 1 sheet. June 2, 1961 . Room 1033, GSA Bldg., Washington, D. C.; Bldg. 25, Federal Center, Denver, Colo.; 345 Middlefield Rd., Menlo Park, Calif.; Brooks Memorial Bldg., College, Alaska; Room 117, Federal Bldg., Juneau, Alaska; State Div. of Mines and Minerals, State Capitol Bldg., Juneau, Alaska; Room 503, Cordova Bldg., Anchorage, Alaska; Room 232, Appraisers Bldg., San Francisco, Calif.; Room 1031, Bartlett Bldg., Los Angeles, Calif.; South 157 Howard St.. Spokane, Wash.; Room 468, New Custom House, Denver, Colo.; Room 602, Thomas Bldg., Dallas, Tex.

101. Miller, D. J., Geology of the Malaspina district, Gulf of Alaska Tertiary province, Alaska: 2 sheets. June 2, 1961. Room 1033, GSA Bldg., Washington, D. C.; Bldg. 25, Federal Center, Denver, Colo.; 345 Middlefield Rd., Menlo Park, Calif.; Brooks Memorial Bldg., College, Alaska; Room 117, Federal Bldg., Juneau, Alaska; State Div. of Mines and Minerals, State Capitol Bldg., Juneau, Alaska; Room 503, Cordova Bldg., Anchorage, Alaska; Room 232, Appraisers Bldg., San Francisco, Calif.; Room 1031, Bartlett Bldg., Los Angeles, Calif.; South 157 Howard St. Spokane, Wash.; Room 468, New Custom House, Denver, Colo.; Room 602, Thomas Bldg., Dallas, Tex.

102. Miller, D. J., Geology of the Yakataga district, Gulf of Alaska Tertiary province, Alaska: 2 sheets. June 2, 1961. Room 1033, GSA Bldg., Washington, D. C.; Bldg. 25, Federal Center, Denver, Colo.; 345 Middlefield Rd., Menlo Park, Calif.; Brooks Memorial Bldg., College, Alaska; Room 117, Federal Bldg., Juneau, Alaska; State Div. of Mines and Minerals, State Capitol Bldg., Juneau, Alaska; Room 503, Cordova Bldg., Anchorage, Alaska; Room 232, Appraisers Bldg., San Francisco, Calif.; Room 1031, Bartlett Bldg., Los Angeles, Calif.; South 157 Howard St., Spokane, Wash.; Room 468, New Custom House, Denver, Colo.; Room 502, Thomas Bldg., Dallas, Tex.

103. Miller, D. J., Geology of the Yakutat district, Gulf of Alaska Tertiary province, Alaska: 1 sheet. June 2, 1961: Room 1033, GSA Bldg., Washington, D. C.; Bldg. 25, Federal Center, Denver, Colo.; 345 Middlefield Rd., Menlo Park, Calif.; Brooks Memorial Bldg., College, Alaska; Room 117, Federal Bldg., Juneau, Alaska; State Div. of Mines and Minerals, State Capitol Bldg., Juneau, Alaska; Room 503, Cordova Bldg., Anchorage, Alaska; Room 232, Appraisers Bldg., San Francisco, Calif.; Room 1031, Bartlett Bldg., Los Angeles, Calif.; South 157 Howard St., Spokane, Wash.; Room 468, New Custom House, Denver, Colo.; Room 602, Thomas Bldg., Dallas, Tex.

104. Miller, R. E., Land subsidence in the Los BanosKettleman City area, 1957-59: 1 map. July 7, 
1961. Room 8024, Federal and U.S. Court House Bldg., 650 Capitol Ave., Sacramento, Calif.; Room 1242-G, GSA Bldg., Washington, D. C.

105. Milton, D. J., Geology of the Old Speck Mountain quadrangle, Maine: 190 p., 2 pl., 16 figs., 53 photographs. Sept. 12, 1961. Room 1033, GSA Bldg., Washington, D. C.; Office of the State Geologist, State House, Augusta, Maine.

106. Moore, J. E., Records of wells, test holes, and springs in the Nevada Test Site and surrounding area: 22 p., 1 fig. July 31, 1961. Room 1033, GSA Bldg., Washington, D. C.; Bldg. 25, Federal Center, Denver, Colo.; 345 Middlefield Rd., Menlo Park, Calif.; Room 232, Appraisers Bldg., San Francisco, Calif.; Room 1031, Bartlett Bldg., Los Angeles, Calif.; Room 437, Federal Bldg., Salt Lake City, Utah; Room 279, Geology Bldg., Univ. of New Mexico, Albuquerque, N. Mex.; Library, Mackay School of Mines, Univ, of Nevada, Reno, Nev.

107. Mower, R. W., Relation of the deep and shallow artesian aquifers near Lynndyl, Utah: 4 p., 4 figs. July 19, 1961. Room 503-A, Federal Bldg., Salt Lake City, Utah; Room 1242-G, GSA Bldg., Washington, D. C.

108. Moyle, W. R., Jr., Ground-water inventory for 1960, Edwards Air Force Base, California: 44 p., 7 figs. Nov. 16, 1961. 221 Redondo Ave., Long Beach, Calif.; Room 8024, Federal and U.S. Court House Bldg., 650 Capitol Ave., Sacramento, Calif.; Room 1242-G, GSA Bldg., Washington, D. C.

109. Mullens, T. E., and Izett, G. A., Preliminary geologic map of the Paradise quadrangle, Utah: 1 map. Aug. 2, 1961. Room 1033, GSA Bldg., Washington, D. C.; Room 437, Federal Bldg., Salt Lake City, Utah; Room 468, New Custom House, Denver, Colo.

110. Mullineaux, D. R., Geology of the Renton, Auburn, and Black Diamond quadrangles, Washington: 202 p., 3 pl., 16 figs. Sept. 22, 1961. Room 1033, GSA Bldg., Washington, D. C.

111. Neal, D. W., Review of waterpower withdrawals in Middle Fork Willamette River Basin, Oregon: 19 p., 1 fig. July 15, 1961. Room 1033, GSA Bldg., Washington, D. C.; 1002 N. E. Holladay St., Portland, Oreg.

112. Olson, J. C., and Adams, J. W., Thorium and rare earths in the United States: 1 map. Dec. 26, 1961. Room 1033, GSA Bldg., Washington, D. C.

113. Orkild, P. P., Geologic map of Tippipah Spring quadrangle, Nye County, Nevada: 1 map with explanation and cross-sections. Aug. 23, 1961. Room 1033, GSA Bldg., Washington, D. C.; Bldg. 25, Federal Center, Denver, Colo.; 345 Middlefield Rd., Menlo Park, Calif.; Mercury, Nev.; Room 232, Appraisers Bldg., San Francisco, Calif.; Room 1031, Bartlett Bldg., Los Angeles, Calif.; Room 437, Federal Bldg., Salt Lake City, Utah; Room 279, Geology Bldg., Univ. of New Mexico, Albuquerque, N. Mex.; Library, Mackay School of Mines, Univ. of Nevada, Reno, Nev.

114. Orkild, P. P., and Pomeroy, J. S., Geologic reconnaissance of the Topopah Spring and Timber Mountain quadrangles, Nye County, Nevada: 20 p., 2 figs., 1 table. Aug. 23, 1961. Room 1033, GSA Bldg., Washington, D. C.; Bldg. 25, Federal Center, Denver, Colo.; 345 Middlefield Rd., Menlo Park, Calif.; Room 232, Appraisers Bldg., San Francisco, Calif.; Room 1031, Bartlett Bldg., Los Angeles, Calif.; Room 437, Federal Bldg., Salt Lake City, Utah; Room 279, Geology Bldg., Univ. of New Mexico, Albuquerque, N. Mex.; Library, Mackay School of Mines, Univ. of Nevada, Reno, Nev.

115. O'Sullivan, R. B., Geologic map of the Cedar Mesa-Boundary Butte area, San Juan County, Utah: 1 geol. map and section with explanations, 11 p. of wells drilled. May 17, 1961. Room 1033, GSA Bldg., Washington, D. C.; Bldg. 25, Federal Center, Denver, Colo.; 345 Middlefield Rd., Menlo Park, Calif.; Room 437, Federal Bldg., Salt Lake City, Utah; Bureau of Indian Affairs, Window Rock, Ariz.

116. Page, R. W., Ground-water conditions during 1959 at the Naval Air Missile Test Center, Point Mugu, California: 32 p., 5 figs. Jan. 10, 1961. 221 Redondo Ave., Long Beach, Calif.; Room 8024, Federal and U.S. Court House Bldg., 650 Capitol Ave., Sacramento, Calif.; Room 1242-G, GSA Bldg., Washington,' D. C.

117. Page, R. W., Ground-water conditions during $\mathbf{1 9 6 0}$ at the U.S. Naval Air Station, Point Mugu, California: 58 p., 5 figs. Nov. 9, 1961. 221 Redondo Ave., Long Beach, Calif.; Room 8024, Federal and U.S. Court House Bldg., 650 Capitol Ave., Sacramento, Calif.; Room 1242-G, GSA Bldg., Washington, D. C.

118. Page, R. W., Test-well drilling, San Nicolas Island, California: 26 p., 2 figs. Aug. 7, 1961. 221 Redondo Ave., Long Beach, Calif.; Room 8024, Federal and U.S. Court House Bldg., 650 Capitol Ave., Sacramento, Calif.; Room 1242-G, GSA Bldg., Washington, D. C.

119. Peterson, D. W., Dacitic ash-flow sheet near Superior and Globe, Arizona: 130 p., 7 pl., 32 figs., 6 tables. Apr. 3, 1961. Room 1033, GSA Bldg., Washington, D. C.

120. Pluhowski, E. J., and Kantrowitz, I. H., Hydrology of the Babylon-Islip area, Suffolk County, Long Island, New York: 118 p., 38 figs. Oct. 27, 1961. 1505 Kellum Place, Mineola, N. Y.; Room 1242-G, GSA Bldg., Washington, D. C.

121. Rabon, J. W., Flow duration of Georgia streams: 326 p., 6 figs. Sept. 28, 1961. Room 609, 805 Peachtree Street Bldg., Atlanta, Ga.; Room 1033, GSA Bldg., Washington, D. C.

122. Randall, A. D., Ground-water basic data, Farmington-Granby area, Connecticut: 95 p. May 17, 1961 . Room 204; P. O. Bldit. Midaldewn, 
Conn.; Room 1242-G, GSA Bldg., Washington, D. C.

123. Rantz, S. E., Flow of springs and small streams in the Tecolote tunnel area of Santa Barbara County, Calif.: 282 p., 9 figs., 1 map. Feb. 16, 1961. Room 1003, Bartlett Bldg., Los Angeles, Calif.; Room 2236, GSA Bldg., Washington, D. C.

124. Reeves, R. G., Geologic map and sections of the Andrade mine, Monlevade quadrangle, Minas Gerais, Brazil: 1 map. Feb. 20, 1961. Room 1033, GSA Bldg., Washington, D. C.; 345 Middlefield Rd., Menlo Park, Calif.; Dept. Nacional da Producâao Mineral, Rio de Janeiro and Belo Horizonte, Brazil.

125. Reeves, R. G., Geologic map of the Monlevade quadrangle, Minas Gerais, Brazil: 1 map. Feb. 20, 1961. Room 1033, GSA Bldg., Washington, D. C.; 345 Middlefield Rd., Menlo Park, Calif.; Dept. Nacional da Produço Mineral, Rio de Janeiro and Belo Horizonte, Brazil.

126. Reeves, R. G., Geologic map of the Rio Piracicaba quadrangle, Minas Gerais, Brazil: 1 map. Feb. 20, 1961. Room 1033, GSA Bldg., Washington, D. C.; 345 Middlefield Rd., Menlo Park, Calif.; Dept. Nacional da Produçăo Mineral, Rio de Janeiro and Belo Horizonte, Brazil.

127. Repenning, C. A., Geologic summary of the Central Valley of California, with reference to disposal of liquid radioactive waste: 69 p., 14 figs. Feb. 28, 1961. Room 1033, GSA Bldg., Washington, D. C.; Bldg. 25, Federal Center, Denver, Colo.; 345 Middlefield Rd., Menlo Park, Calif.; Room 232, Appraisers Bldg., San Francisco, Calif.; Room 1031, Bartlett Bldg., Los Angeles, Calif.; Room 437, Federal Bldg., Salt Lake City, Utah; Room 468, New Custom House, Denver, Colo.; Room 602, Thomas Bldg., Dallas, Tex.; South 157 Howard St., Spokane, Wash.; Room 279, Geology Bldg., Univ. of New Mexico, Albuquerque, N. Mex.; California Div, of Mines, Ferry Bldg., San Francisco, Calif.

128. Riley, F. S., and Bader, J. S., Data on water wells on Marine Corps Base, Twentynine Palms, California: 18 p., 2 figs. May 22, 1961. 2929 Fulton Ave., Sacramento, Calif.;- 221 Redondo Ave., Long Beach, Calif.; Room 1242-G, GSA Bldg., Washington, D. C.

129. Robison, J. H., The geohydrologic appraisal of the Imperial Valley, Arizona: 6 p. Nov. 17, 1961. 16 W. 2d St., Yuma, Ariz.; Room 1242-G, GSA Bldg., Washington, D. C.

130. Rogers, C. L., and Jaster, M. C., Titanium in the United States: 1 map. Dec. 26, 1961. Room 1033, GSA Bldg., Washington, D. C.

131. Sammel, E. A., and Baker, J. A., Records and logs of selected wells and test holes, chemical analyses of water, and water levels in observation wells in the lower Ipswich River basin, Massachusetts: 153 p., 2 figs. Nov. 16, 1961. Room 847, Oliver Bldg., 141 Milk St., Boston, Mass.; Room 1242-G, GSA Bldg., Washington, D. C.
132. Sample, R. D., and Albee, H. F., Claim map, Anderson Mesa quadrangle, Montrose and San Miguel Counties, Colorado: 1 map. Jan. 6, 1961. Room 1033, GSA Bldg., Washington, D. C.; Bldg. 25, Federal Center, Denver, Colo.

133. Sample, R. D., and Albee, H. F., Claim map, Atkinson Creek quadrangle, Montrose County, Colorado: 1 map. Jan. 6, 1961. Room 1033, GSA Bldg., Washington, D. C.; Bldg. 25, Federal Center, Denver, Colo.

134. Sample, R. D., and Albee, H. F., Claim map, Bull Canyon quadrangle, Montrose and San Miguel Counties, Colorado: 1 map. Jan. 6, 1961. Room 1033, GSA Bldg., Washington, D. C.; Bldg. 25. Federal Center, Denver, Colo.

135. Sample, R. D., Albee, H. F., and Stephens, H. G., Claim map, Bull Canyon quadrangle, Montrose County, Colorado (revised): 1 map. Jan. 6, 1961. Room 1033, GSA Bldg.. Washington, D. C.; Bldg. 25, Federal Center, Denver, Colo.

136. Sample, R. D., and Albee, H. F., Claim map, Calamity Mesa quadrangle, Colorado: 1 map. Jan. 6, 1961. Room 1033, GSA Bldg., Washington, D. C.; Bldg. 25, Federal Center, Denver, Colo.

137. Sample, R. D., and Albee, H. F., Claim map, Davis Mesa quadrangle, Montrose County, Colorado: 1 map. Jan. 6, 1961. Room 1033, GSA Bldg., Washington, D. C.; Bldg. 25, Federal Center, Denver, Colo.

138. Sample, R.- D., and Albee, H. F., Claim map, Egnar quadrangle, San Miguel County, Colorado: 1 map. Jan. 6, 1961. Room 1033, GSA Bldg., Washington, D. C.; Bldg. 25, Federal Center, Denver, Colo.

139. Sample, R. D., and Albee, H. F., Claim map, Gateway quadrangle, Mesa County, Colorado: 1 map. Jan. 6, 1961. Room 1033, GSA Bldg., Washington, D. C.; Bldg. 25, Federal Center, Denver, Colo.

140. Sample, R. D., and Albee, H. F., Claim map, Gypsum Gap quadrangle, San Miguel County, Colorado: 1 map. Jan. 6, 1961. Room 1033, GSA Bldg., Washington, D. C.; Bldg. 25, Federal Center, Denver, Colo.

141. Sample, R. D., Albee, H. F., and Stephens, H. G., Claim map, Hamm Canyon quadrangle, San Miguel County, Colorado: 1 map. Jan. 6, 1961. Room 1033, GSA Bldg., Washington, D. C.; Bldg. 25, Federal Center, Denver, Colo.

142. Sample, R. D., Albee, H. F., and Stephens, H. G., Claim map, Horse Range Mesa quadrangle, San Miguel County, Colorado: 1 map. Jan. 6, 1961. Room 1033, GSA Bldg., Washington, D. C.; Bldg. 25, Federal Center, Denver, Colo.

143. Sample, R. D., and Albee, H. F., Claim map, Joe Davis Hill quadrangle, San Miguel County, Colorado: 1 map. Jan. 6, 1961. Room 1033, GSA Bldg., Washington, D. C.; Bldg. 25, Federal Center, Denver, Colo. 
144. Sample, R. D., and Albee, H. F., Claim map, Juanita Arch quadrangle, Mesa County, Colorado: 1 map. Jan. 6, 1961. Room 1033, GSA Bldg., Washington, D. C.; Bldg. 25, Federal Center, Denver, Colo.

145. Sample, R. D., and Albee, H. F., Claim map, Naturita NW quadrangle, Montrose and San Miguel Counties, Colorado: 1 map. Jan. 6, 1961. Room 1033, GSA Bldg., Washington, D. C.; Bldg. 25, Federal Center, Denver, Colo.

146. Sample, R. D., and Albee, H. F., Claim map, Paradox quadrangle, Montrose County, Color: ado: 1 map. Jan. 6, 1961. Room 1033, GSA Bldg., Washington, D. C.; Bldg. 25, Federal Center, Denver, Colo.

147. Sample, R. D., and Albee, H. F., Claim map, Pine Mountain quadrangle, Mesa County, Colorado: 1 map. Jan. 6, 1961. Room 1033, GSA Bldg., Washington, D. C.; Bldg. 25, Federal Center, Denver, Colo.

148. Sample, R. D., and Albee, H. F., Claim map, Red Canyon quadrangle, Montrose County, Colorado: 1 map. Jan. 6, 1961. Room 1033, GSA Bldg., Washington, D. C.; Bldg. 25, Federal Center, Denver, Colo.

149. Sample, R. D., and Albee, H. F., Claim map, Roc Creek quadrangle, Montrose County, Colorado: 1 map. Jan. 6, 1961. Room 1033, GSA Bldg., Washington, D. C.; Bldg. 25, Federal Center, Denver, Colo.

150. Sample, R. D., and Albee, H. F., Claim map, Uravan quadrangle, Montrose County, Colorado: 1 map. Jan. 6, 1961. Room 1033, GSA Bldg., Washington, D. C.; Bldg. 25, Federal Center, Denver, Colo.

151. Schoellhamer, J. E., and Yerkes, R. F., Preliminary qeologic map of the coastal part of the Malibu Beach quadrangle, Los Angeles County, California: 1 map, 1 explanation. Sept. 6, 1961. Room 1033, GSA Bldg., Washington, D. C.; Bldg. 25, Federal Center, Denver, Colo.; 345 Middlefield Rd., Menlo Park, Calif.; Room 232, Appraisers Bldg., San Francisco, Calif.; Room 1031 , Bartlett Bldg., Los Angeles, Calif.; Office of Los Angeles County Engineer, Room 721 , County Engineer Bldg., 108 W. 2d St., Los Angeles, Calif.; California Div. of Mines, Ferry Bldg., San Francisco, Calif.; California Div. of Mines, State Office Bldg. No. 2, 107 South Brcadway, Los Angeles, Calif.

152. Schoff, S. L., and Winograd, I. J., Hydrologic significance of six core holes in carbonate rocks of the Nevada Test Site: 97 p., 4 figs. Aug. 21, 1961. Room 1033, GSA Bldg., Washington, D. C.; Bldg. 25, Federal Center, Denver, Colo.; 345 Middlefield Rd., Menlo Park, Calif.; Room 232, Appraisers Bldg., San Francisco, Calif.; Room 1031, Bartlett Bldg., Los Angeles, Calif.; Room 437, Federal Bldg., Salt Lake City, Utah; Room 279, Geology Bldg., Univ. of New Mexico, Albuquerque, N. Mex.; Library, Mackay School of Mines, Univ. of Nevada, Reno, Nev.
.153. Schultz, L. G., Preliminary report on the geology and mineralogy of clays on the Pine Ridge Indian Reservation, South Dakota, with a chapter on usability tests, by H. P. Hamlin: 60 p., 1 pl., 2 tables. Nov. 3, 1961 . Room 1033, GSA Bldg., Washington, D. C.; Bldg. 25, Federal Center, Denver, Colo.; 345 Middlefield Rd., Menlo Park, Calif.; Room 468, New Custom House, Denver, Colo.; Office of the State Geologist, State University, Vermillion, S. Dak.

154. Shawe, F. R., Reeves, R. G., and Kral, V. E., Iron ore deposits of northern Nevada: 83 p., 8 pl., 10 figs., 8 tables. Jan. 27, 1961. Room 1033, GSA Bldg., Washington, D. C.; 345 Middlefield Rd., Menlo Park, Calif.; Library, Mackay School of Mines, Univ. of Nevada, Reno, Nev.

155. Shride, A. F., Some aspects of younger Precambrian geology in southern Arizona: 387 p., 16 figs., 5 tables. Sept. 22, 1961. Room 1033, GSA Bldg., Washington, D. C.

156. Simons, F. S., Geologic map and sections of the Klondyke quadraingle, Arizona: 1 map and sections, plus explanation. May 8, 1961. Room 1033, GSA Bldg., Washington, D. C.; Bldg. 25, Federal Center, Denver, Colo.; 345 Middlefield Rd., Menlo Park, Calif.; Room 437, Federal Bldg., Salt Lake City, Utah; Room 232, Appraisers Bldg., San Francisco, Calif.; Room 1031, Bartlett Bldg., Los Angeles, Calif.; Room 468, New Custom House, Denver, Colo.; Office of the Director, College of Mines, Arizona Bureau of Mines, Univ. of Arizona, Tucson, Ariz.

157. Sisco, H. G., and Luscombe, R. W., Records of wells and water-level fluctuations in the Aberdeen-Springfield area, Bingham and Power Counties, Idaho, in 1960: 16 p., 14 figs. Aug. 21, 1961. 914 Jefferson St., Boise, Idaho; Room 1242-G, GSA Bldg., Washington, D. C.

158. Smith, W. C., Borates in the United States: 1 map. Dec. 26, 1961. Room 1033, GSA Bldg., Washington, D. C.

159. Staatz, M. H., and Carr, W. J., Geologic map of the Thomas and Dugway Ranges, Juab and Tooele Counties, Utah: 1 map and explanation. Jan. 6, 1961. Room 1033, GSA Bldg., Washington, D. C.; Bldg. 25, Federal Center, Denver, Colo.; Room 437, Federal Bldg., Salt Lake City, Utah.

160. Stewart, H. G., Map of Polk County, Florida, showing contours on the piezometric surface of the Floridan aquifer: 1 map. July 6, 1961. Gunter Bldg., Corner of Tennessee and Woodward Sts., Tallahassee, Fla.; Room 1242-G, GSA Bldg., Washington, D. C.

161. Stewart, J. H., Stratigraphy and origin of the Chinle formation (Upper Triassic) of the Colorado Plateau: 196 p., 48 figs., 2 tables. Apr. 24, 1961. Room 1033, GSA Bldg., Washington, D. C.

162. Thayer, T. P., and Miller, M. H., Chromite in the United States: 1. map. Dec. 26, 1961. Room 1033, GSA Bldg., Washington, D. C. 
163. Thomas, D. M., Extent and frequency of inundation of flood plain in vicinity of Somerville and Manville, N. J.: 24 p., 8 figs. Mar. 20, 1961. Room 228, Federal Bldg., Trenton, N. J.; Room 2236, GSA Bldg., Washington, D. C.

164. Tolozko, L. R., Preliminary isoradioactivity map of the Tordilla Hill-Deweesville area, Karnes County, Texas: 1 map. Room 1033, GSA Bldg., Washington, D. C.; Bldg. 25, Federal Center, Denver, Colo.; 345 Middlefield Rd., Menlo Park, Calif.; Room 602; Thomas Bldg., Dallas, Tex.; Balcones Research Center, Austin, Tex.

165. Tourtelot, H. A., and Schultz, L. G., Core from the Irish Creek well, Ziebach County, South Dakota: 20 p., 2 figs. Room 1033, GSA Bldg., Washington, D. C.; Bldg. 25, Federal Center, Denver, Colo.; 345 Middlefield Rd., Menlo Park, Calif.; South Dakota Geol. Survey, State Univ., Vermillion, S. Dak.

166. Waldron, H. H., Geology of the Des Moines quadrangle; Washington: 1 map and explanation. Jan. 27, 1961. Room 1033, GSA Bldg., Washington, D. C.; Bldg. 25, Federal Center, Denver, Colo.; 345 Middlefield Rd., Menlo Park, Calif.; South 157 Howard St., Spokane, Wash.; 3020 South 38th St., Tacoma, Wash.; Div. of Mines and Geology, Room 335, General Admin. Bldg., Olympia, Wash.

167. Waldron, H. H., Geology of the Poverty Bay quadrangle, Washington: 1 map and explanation. Jan. 27, 1961. Room 1033, GSA Bldg., Washington, D. C.; Bldg. 25, Federal Center, Denver, Colo.; 345 Middlefield Rd., Menlo Park, Calif.; South 157 Howard St., Spokane, Wash.; 3020 South 38th St., Tacoma, Wash.; Div. of Mines and Geology, Room 335, General Admin. Bldg., Olympia, Wash.

168. Waldron, H. H., Leisch, B. A., Mullineaux, D. R., and Crandell, D. R., Preliminary geologic map of Seattle and vicinity, Washington: 2 maps, 1 explanation, 1 chart. Dec. 5, 1961. Room 1033, GSA Bldg., Washington, D. C.; Bldg. 25, Federal Center, Denver, Colo.; 345 Middlefield Rd., Menlo Park, Calif.; 3020 South 38th St., Tacoma, Wash.; South 157 Howard St., Spokane, Wash.; Room 232, Appraisers Bldg., San Francisco, Calif.; Room 1031, Bartlett Bldg., Los Angeles, Calif.; State Div, of Mines and Geology, Room 335, General Admin. Bldg., Olympia, Wash.

169. Weber, F. R., Reconnaissance engineering geology for selection of highway route from Talkeetna to McGrath, Alaska: 1 map (2 sheets). Dec. 5,
1961. Room 1033, GSA Bldg., Washington, D. C.; Bldg. 25, Federal Center, Denver, Colo.; 345 Middlefield Rd., Menlo Park, Calif.; Brooks Memorial Bldg., College, Alaska; Room 111, Federal Bldg., Juneau, Alaska; Room 503, Cordova Bldg., Anchorage, Alaska; State Div. of Mines and Minerals, State Capitol Bldg., Juneau, Alaska; Room 232, Appraisers Bldg., San Francisco, Calif.; Room 1031, Bartlett Bldg., Los Angeles, Calif.; South 157 Howard St., Spokane, Wash.; Room 468, New Custom House, Denver, Colo.; Room 602, Thomas Bldg., Dallas, Tex.; Alaskan Geology Branch, 345 Middlefield Rd., Menlo Park, Calif.

170. Wetterhall, W. S., Stewart, H. G., Jr., and Meyer, F. W., Map showing piezometric surface of the Floridan aquifer in Pasco County and adjacent areas, Florida: 1 map. June 27, 1961. Gunter Bldg., Corner of Tennessee and Woodward Sts., Tallahassee, Fla.; Room 1242-G, GSA Bldg., Washington, D. C.

171. White, D. E., Antimony in the United States: 1 map. Dec. 26, 1961. Room 1033, GSA Bldg., Washington, D. C.

172. Wiitala, S. W., Some aspects of the effect of urban and suburban development upon runoff: 28 p., 9 figs. Oct. 30, 1961. Room 407, Capitol Savings and Loan Bldg., Lansing, Mich.; 345 Middlefield Rd., Menlo Park, Calif.; Bldg. 25, Federal Center, Denver, Colo.; Room 1033, GSA Bldg., Washington, D. C.

173. Wilson, K. V., Floods of 1958 in Mississippi: 35 p., 12 figs. May 23, 1961. 402 High St., Jackson, Miss.; Room 2236, GSA Bldg., Washington, D. C.

174. Withington, C. F., Gypsum and anhydrite in the United States: 1 map. Dec, 26, 1961. Room 1033, GSA Bldg., Washington, D. C.

175. Yates, R. G., Geology of part of the Boundary and Spirit quadrangles, Stevens County, Washington: 1 map. Room 1033, GSA Bldg., Washington, D. C.; Bldg. 25, Federal Center, Denver, Colo.; 345 Middlefield Rd., Menlo Park, Calif.; South 157 Howard St., Spokane, Wash.; State Div. of Mines and Geology, Room 335, General Admin. Bldg., Olympia, Wash.

176. Young, R. A., Hydrogeologic evaluation of streamflow records in the Rogue River basin, Oregon: 119 p., 2 pls., 53 figs. July 7, 1961. Federal Bldg., 1002 N. E. Holladay St., Portland, Oreg.; Room 1242-G, GSA Bldg., Washington, D. C. 

Alaska:

Chariot, Project, site.....

Katalla district .

Ladd Air Force Base, well data..........

Lake Chakachamna, potential waterpower ...

Lituya district .................

Malaspina district....

Pybus-Gambier area

Snow River, Nellie Juan Lake, and Lost

Lake, Kenai Peninsula, waterpower

resources _............

Talkeetna to McGrath, highway route ......

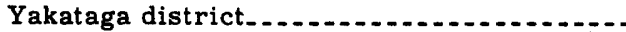

Yakutat district

Yukon, Lower................................

Antimony in the United States.....

Arizona:

Imperial Valley, geohydrology . . .

Klondyke quadrangle.

Navajo Indian Reservation, geologic and water maps.........................

Precambrian geology in southern Arizona...

Salton Sea, evaporation, energy-budget method.

Superior and Globe, dacitic ash-flow sheet near

Asbestos in the United States .

Bismuth in the United States.......

Borates in the United States

Brazil:

Andrade mine, Monlevade quadrangle.......

Monlevade quadrangle ....................

Rio Piracicaba quadrangle ....

California:

Central Valley ..........

Edwards Air Force Base, ground-water inventory, 1960

Los Banos-Kettleman City area, land

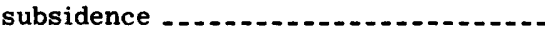

Malibu Beach quadrangle, coastal part......

Pedernales, ground-water availability .......

Point Arguello, ground-water conditions -..--

Point Mugu, ground-water conditions, 1959.-

Point Mugu, ground-water conditions, 1960..

San Nicholas Island, test-well drilling ......

Santa Barbara County, water levels, $1960 \ldots$

Tecolote tunnel area, streamflow and

springs -

Twentynine Palms, ground-water conditions, 1960

Twentynine Palms, well data .

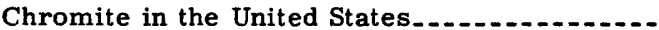

Clays:

Pine Ridge Indian Reservation, South Dakota. Coàl:

Rangely, Colorado...........

Colorado:

Anderson Mesa quadrangle, claim map ..... 132

Atkinson Creek quadrangle, claim map .... - 133

Boulder Creek, floods _._. 77

Bull Canyon quadrangle, claim map ....... 134

Bull Canyon quadrangle, claim map (rev.)._- 135

Calamity Mesa quadrangle, claim map.....- 136

Davis Mesa quadrangle, claim map ........ 137

Egnar quadrangle, claim map._. 138

Frenchy Incline uranium deposit ........ 98

Gateway quadrangle, claim map........ 139
Colorado-Continued

Report

Gypsum Gap quadrangle, claim map ........ 140

Hamm Canyon quadrangle, claim map ..... 141

Horse Range Mesa quadrangle, claim map .. 142

Joe Davis Hill quadrangle, claim map ...... 143

Juanita Arch quadrangle, claim map....... 144

Naturita NW quadrangle, claim map......-. 145

Paradox quadrangle, claim map............ 146

Pine Mountain quadrangle, claim map ...... 147

Rangely coal area

Red Canyon quadrangle, claim map....... 148

Roc Creek quadrangle, claim map......... 149

Uravan quadrangle, claim map.......... 150

Colorado Plateau:

Chinle formation, stratigraphy and origin ...-

Colorado River, lower:

Geohydrologic investigations . . . . . .

Ground-water resources . . . .

Study of hydrology.

Surface-water supply -

Water quality

Water-resources investigation.........

Connecticut:

Farmington-Granby area, ground-water data.

Copper in the United States...

District of Columbia area:

Ground-water and subsurface data .......

Flurida:

Pasco County, ground-water map _._._. 170

Polk County, ground-water map.....-..- 160

Gamma-radioactivity investigations, Nevada

Test Site _.

Gamma-ray logs, utilization of, 1949-1953 ....

Georgia:

Flow duration of streams

Gold in the United States .

Ground water:

Bibliography, general, 1960

Gypsum and anhydrite in the United States .....

Idaho:

Aberdeen-Springfield area, well data, 1960.-

Irwin quadrangle . . .

National Reactor Testing Sta., waste disposal

Snake River plain, western, gravity survey.-

Stewart Flat quadrangle...... Iron:

Carter Creek, Montana

Kelly iron deposit, Montana

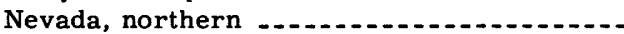

Isostatic deformation of Bonneville shorelines.

Kentucky:

Green River at Munfordville, water quality--

Lake Bonneville, isostatic deformation, shorelines._. 39

Lead in the United States........ 94

Magnesite and brucite in the United States .... 56

Maine:

Attean quadrangle, preliminary geologic ....

Attean and part of Sandy Bay quadrangles, aeromagnetic ......

Bingham and The Forks quadrangles, aeromagnetic .

Gravity data in west-central Maine........

Old Speck Mountain quadrangle

Manganese in the United States .

161 
Massachusetts:

Ipswich River basin, well data

Mercury in the United States .

Michigan basin, disposal of radioactive wastes.

Minnesota:

Chisholm-Dewey Lake area, water levels and surficial geology ....................

Mississippi:

Floods of 1958

Missouri:

Ground-water problems..................

Montana:

Carter Creek iron deposit..............

Glacier National Park, glacier observations.

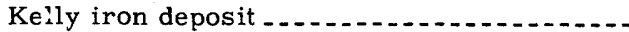

Nebraska:

Uranium in Chadron area

Nevada, northern, iron ore deposits ........

Nevada Test Site:

Core holes, carbonate rocks............

Frenchman and Yucca Valleys, well data...-

Gamma-radioactivity investigations ........

Gold Meadows, Climax, and Dolomite Hills..

Gold Meadows, Tem Piute, and Trappman's

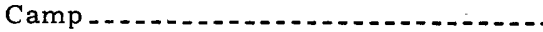

Oak Spring formation, seismic velocities ...

Oak Spring quadrangle .......

Tippipah Spring quadrangle...............

Topopah Spring and Timber Mountain quadrangles - .

U12b tunnel system. ...................

U12e tunnel system.

U15b area, Climax Stock, logs of core holes.

Well and spring records ...............

New Hampshire:

Southeastern, ground-water data

New Jersey:

Flood plain inundation, Somerville-

Manville area...........................

New Mexico:

Northern Lea County, geology and ground

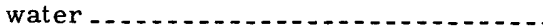

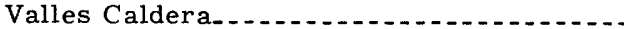

New York:

Babylon-Islip area, hydrology-.............

Big Moose quadrangle, aeromagnetic map...

Bolton, Whitehall, Glens Falls, and Fort Ann quadrangles, aeromagnetic map...

Elizabethtown, Paradox Lake, Port Henry, and Ticonderoga quadrangles, aeromagnetic map. ..................

Long Island, ground-water levels, 1958-59 _.

Lowville quadrangle, aeromagnetic map ....

McKeever quadrangle, aeromagnetic map ...

Number Four quadrangle, aeromagnetic map . . . . .

Old Forge and part of West Canada Lakes quadrangles, aeromagnetic map.......

Port Leyden quadrangle, aeromagnetic map .

Oklahoma:

Beaver Creek basin, ground water ........

Elk Creek basin, ground water..............

Otter Creek basin, ground water ..........

Roosevelt area, ground water...........

Oregon:

McKenzie River Basin, waterpower with-

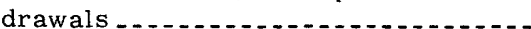

Report
Oregon-Continued

Middle Fork Willamette River basin, waterpower withdrawals . .

Rogue River basin, streamflow. ......... Pennsylvania:

Floods, frequency and magnitude.........

Puerto Rico, preliminary geologic map .......

Pyrophyllite and kyanite in the United States .Radioactive waste:

Central Valley of California ..................

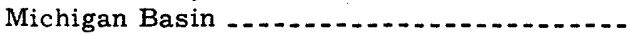

Sedimentary basins of United States .......

South Dakota:

Chadron area, Nebraska-South Dakota ......

Irish Creek well .

Pine Ridge Indian Reservation .

Streamflow:

Effect of urban and suburban development...

Talc and soapstone in the United States .......

Texas:

Tordilla Hill-Deweesville area ........... Thorium and rare earths in the United States.Titanium in the United States ..... Tungsten in the United States Uranium:

Chadron area, Nebraska-South Dakota ......

Epigenetic uranium in United States ........

Frenchy Incline deposit, Colorado......... Utah:

Aspen Grove quadrangle .

Bonneville shorelines, isostatic deformation of

Brighton quadrangle ...................

Cedar Mesa-Boundary Butte area ..........

Ground-water levels, April..............

Ground-water levels, October 1961.......

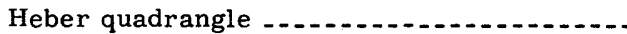

Lisbon Valley area, geochemical studies...-

Lynndyl area, aquifers.............

Orem quadrangle...

Paradise quadrangle......................

Strawberry Valley quadrangle .

Thomas and Dugway Ranges .

Vanadium in the United States . . .

Washington:

Auburn quadrangle

Black Diamond quadrangle . . .

Boundary quadrangle .......

Des Moines quadrangle . . . . . . . . . .

Orting quadrangle ........

Poverty Bay quadrangle...........

Renton quadrangle......

Seattle and vicinity

Spirit quadrangle.....

Sumner quadrangle ..........

Wilkeson quadrangle........

Water measurement:

Current meters, care and rating -......

Wisconsin:

Floods, magnitude and frequency

Streamflow, Wisconsin River, Dekorra ..... Wyoming:

Irwin quadrangle, Idaho-Wyoming - . . - .

Zinc in the United States
Report

111

176

28

24

46

127

40

90

42

165

153

172

32

164

112

130

87

42

29

98

5

39

6

115

49

22

7

83

107

8

109

9

159

110

175

166

35

167

110

168

175

36

37

52

44

45

53

95 